\title{
Factors associated with a prolonged length of stay after acute exacerbation of chronic obstructive pulmonary disease (AECOPD)
}

This article was published in the following Dove Press journal:

International Journal of COPD

20 January 2014

Number of times this article has been viewed

Ying Wang ${ }^{1-3}$

Knut Stavem ${ }^{1-3}$

Fredrik A Dahl'

Sjur Humerfelt ${ }^{4}$

Torbjørn Haugen ${ }^{1,5}$

'Health Services Research Unit,

${ }^{2}$ Department of Pulmonary Medicine, Akershus University Hospital, ${ }^{3}$ Faculty of Medicine, University of Oslo, Lørenskog, Norway; ${ }^{4}$ Department of Pulmonary Medicine, Oslo University Hospital, Aker, ${ }^{5} \mathrm{Clinic}$ for Allergy and Airway Diseases, Oslo, Norway
Correspondence: Ying Wang Health Services Research Unit, Akershus University Hospital, PO Box 95, N-1478 Lørenskog, Norway Tel +47 67968708 , Fax +47 67989900

Email ying.wang@ahus.no
Background: Early identification of patients with a prolonged stay due to acute exacerbation of chronic obstructive pulmonary disease (COPD) may reduce risk of adverse event and treatment costs. This study aimed to identify predictors of prolonged stay after acute exacerbation of COPD based on variables on admission; the study also looked to establish a prediction model for length of stay (LOS).

Methods: We extracted demographic and clinical data from the medical records of 599 patients discharged after an acute exacerbation of COPD between March 2006 and December 2008 at Oslo University Hospital, Aker. We used logistic regression analyses to assess predictors of a length of stay above the 75th percentile and assessed the area under the receiving operating characteristic curve to evaluate the model's performance.

Results: We included 590 patients (54\% women) aged $73.2 \pm 10.8$ years (mean \pm standard deviation) in the analyses. Median LOS was 6.0 days (interquartile range [IQR] 3.5-11.0). In multivariate analysis, admission between Thursday and Saturday (odds ratio [OR] 2.24 [95\% CI 1.60-3.51], $P<0.001$ ), heart failure (OR 2.26, 95\% CI 1.34-3.80), diabetes (OR 1.90, 95\% CI 1.07-3.37), stroke (OR 1.83, 95\% CI 1.04-3.21), high arterial $\mathrm{PCO}_{2}(\mathrm{OR} 1.26$ [95\% CI 1.13-1.41], $P<0.001$ ), and low serum albumin level (OR 0.92 [95\% CI 0.87-0.97], $P=0.001$ ) were associated with a LOS $>11$ days. The statistical model had an area under the receiver operating characteristic curve of 0.73 .

Conclusion: Admission between Thursday and Saturday, heart failure, diabetes, stroke, high arterial $\mathrm{PCO}_{2}$, and low serum albumin level were associated with a prolonged LOS. These findings may help physicians to identify patients that will need a prolonged LOS in the early stages of admission. However, the predictive model exhibited suboptimal performance and hence is not ready for clinical use.

Keywords: exacerbation, COPD, predictors, length of stay, predicting model

\section{Introduction}

Hospitalization for acute exacerbation of chronic obstructive pulmonary disease (AECOPD) is recognized as a major event in the natural history of chronic obstructive pulmonary disease (COPD) due to its negative effect on lung function, survival, risk of readmission, and quality of life. ${ }^{1-6}$ The prolonged length of stay (LOS) may also indicate more fragile patients who need more attention from health personel. ${ }^{7}$ Although only $10 \%-15 \%$ of all patients with COPD will experience severe exacerbations that require hospital admission, expenditures associated with hospitalization represent more than $70 \%$ of all COPD-related medical care costs. ${ }^{8}$

Until now, no established predictors of prolonged LOS are available, and there is no international accepted norm of how long a hospitalization due to AECOPD 
should be. This is illustrated by the wide range of reported LOS (3-16 days). ${ }^{9,10}$ In the absence of a published definition for prolonged LOS, we defined a LOS longer than the 75th percentile as prolonged LOS in this article, which is in line with other studies. ${ }^{11-13}$

A limited number of studies have published a heterogeneous set of variables that are associated with long hospital stay for AECOPD. For example, social and demographic data, ${ }^{14,15}$ clinical variables, ${ }^{10,16-18}$ comorbidities, ${ }^{11,19-22}$ number and type of drugs used on admission, ${ }^{11}$ and day of the week of the admission ${ }^{16}$ are among the risk factors for long LOS. There is a lack of consistency in identified predictors of long LOS, which makes it difficult to determine which prognostic factors are most important. To the best of our knowledge, there are no established statistical models for predicting LOS based on variables collected at the time of hospital admission for AECOPD. Thus, increased knowledge about predictors of prolonged LOS and earlier prediction of LOS might contribute to better patient treatment, optimal discharge planning, and shortening of the LOS, which will ultimately lead to cost savings for hospitals.

The primary objective of this retrospective study was to identify predictors of prolonged LOS in patients hospitalized for AECOPD. A secondary objective was to develop a predictive model for LOS based on available variables obtained at the time of admission for AECOPD.

\section{Material and methods}

\section{Patients}

The study included all patients with a discharge diagnosis of COPD from March 2006 until December 2008, after introduction of electronic medical records at Oslo University Hospital, Aker. Based on the International Statistical Classification of Disease and Related Health Problems, 10th Revision (ICD-10) ${ }^{23}$, we included both patients with COPD (J43 or J44) as the main diagnosis, and patients with respiratory failure (J96) or pneumonia (J12-J18) as the main diagnosis and COPD (J43 or J44) as a secondary diagnosis. Although patients with different main diagnoses might have different LOS, we decided not to split the sample to keep it as big as possible. Another reason was that the diagnoses were set at the time of discharge and were therefore not available at the time of admission and could not be used as a predictor in this study. We used the diagnosis only as a criterion for patient inclusion. We chose to include the first hospitalization of all consecutive patients hospitalized for AECOPD in this period, regardless of number of previous admissions. In total, 599 patients were included.

\section{Medical record review and variables}

We extracted the following data from the computerized medical record at the time of hospital admission: demographic data, comorbidities, use of long-term oxygen therapy, and clinical data obtained in the emergency department (Table 1). We registered a history of any of the following conditions as comorbidities: ischemic heart disease, cardiac arrhythmias, chronic heart failure, pulmonary heart disease or pulmonary hypertension, psychiatric disorder, general atherosclerosis, stroke, diabetes mellitus, other neurologic disorders than cerebral insult, any malignancy ever, osteoporosis, renal failure, or abuse of drugs or alcohol. We created a summary variable using the total number of these comorbidities, which again was categorized as 0 , or $\geq 1$ comorbidity. We analyzed the impact of both individual comorbidities and the dichotomized comorbidity groups, eg, with or without registered comorbidities, on LOS.

We also extracted the value of the forced expiratory volume in 1 second $\left(\mathrm{FEV}_{1}\right)$ determined at the time closest to that of the admission, LOS, number of COPD-related admissions during the previous 12 months before the current admission, and the day of the week of admission. We then dichotomized weekdays into Thursday-Saturday and Sunday-Wednesday. We analyzed the impact of both individual weekday and dichotomized weekdays on LOS. The reason for dichotomizing weekday was because we considered that the natural time of discharge for those admitted between Thursday and Saturday would be before the following weekend, and thus

Table I Descriptive statistics for patients hospitalized for COPD and included in the final analysis $(n=590)$, mean \pm SD or number (\%)

\begin{tabular}{|c|c|}
\hline Variables & Values \\
\hline Age, years & $73.2 \pm 10.7$ \\
\hline Sex, female & $319(54)$ \\
\hline Mean arterial blood pressure, $\mathrm{mmHg}$ & $104 \pm 16.4$ \\
\hline Pulse rate, beats/min & $95 \pm 21.7$ \\
\hline $\mathrm{PaCO}_{2}, \mathrm{kPa}$ & $5.9 \pm 1.6$ \\
\hline Serum albumin level, $g / L$ & $38.8 \pm 4.6$ \\
\hline $\mathrm{FEV}_{1}, \mathrm{l} / \mathrm{s}$ & $1.0 \pm 0.50$ \\
\hline Patients living in an institution & $61(10)$ \\
\hline Long-term oxygen therapy & $42(7)$ \\
\hline X-ray findings consistent with pneumonia & $176(30)$ \\
\hline At least one comorbidity & $498(84)$ \\
\hline Psychiatric diagnosis & $87(15)$ \\
\hline At least one COPD-related admission* & III (I9) \\
\hline Admission Thursday-Saturday & $237(40)$ \\
\hline
\end{tabular}

Note: *At least one COPD-related admission within 12 months prior to index admission.

Abbreviations: COPD, chronic obstructive pulmonary disease; SD, standard deviation; $\mathrm{PaCO}_{2}$, partial pressure of carbon dioxide in arterial blood; $\mathrm{FEV}_{1}$, forced expiratory volume in I second. 
that they might have a higher risk of a prolonged stay. In the final multivariate analysis, we used only dichotomized weekdays in order to reduce the number of variables in the prediction model. A COPD-related admission was defined by discharge diagnoses from previous admissions using the same criteria as for the current admission.

This study was considered as a quality assessment study by the Regional Committee for Medical and Health Research Ethic, South East Norway (S-09079d, 2009/123) and approved by the local Privacy Ombudsman for Clinical Research, Oslo University Hospital (2011-12102). The later also approved that written consent from each patient was not necessary.

\section{Statistical analysis}

Based on a literature review, we analyzed how 14 variables from our data set were related to LOS (Table 1). Descriptive statistics are expressed as mean \pm standard deviation (SD) or median (interquartile range [IQR]) values for continuous data, and number (\%) for categorical data. Missing values for $\mathrm{FEV}_{1}$, serum albumin level, and arterial carbon dioxide tension $\left(\mathrm{PaCO}_{2}\right)$ were imputed from the other known variables using linear regression analysis; we did not impute missing values for the other variables. Patients with missing values after this procedure were excluded from the final analysis. We used univariate and multivariate logistic regression analyses to assess predictors of a LOS $>11$ days (corresponding to $>75$ th percentile). The multivariate analysis initially included variables with $P \leq 0.25$ in the univariate analysis. We then manually removed variables one by one while watching changes in the coefficients in order to arrive at a final parsimonious model. We chose a significance level of $5 \%$. Stata software (version 10.1) was used for statistical analysis (Stata Corp, College Station, TX, USA).

In an attempt to establish a statistical model to predict whether or not a patient would be hospitalized for longer than the 75th percentile, receiver operating characteristic (ROC) analysis was performed for independent predictors of a long LOS as determined by multivariate logistic regression analysis.

\section{Results \\ Study subjects}

Of 599 patients discharged after AECOPD, nine were excluded from further analysis due to missing data after our imputation of $\mathrm{FEV}_{1}$, serum albumin level, and $\mathrm{PaCO}_{2}$ values. The final sample of 590 patients had a mean age of $73.2 \pm 10.8$ years and $54 \%$ female. The mean LOS was $8.9 \pm 9.7$ days, with a median of 6 days (IQR 3.5-11.0 days). Descriptive statistics for the patients are presented in Table 1.

In univariate analysis, age, $\mathrm{FEV}_{1}$, COPD-related admission during the previous 12 months before the current admission, admission between Thursday and Saturday (here under, Thursday and Saturday), high $\mathrm{PaCO}_{2}$, high pulse rate, low serum albumin level, and having one of a selection of comorbidities (ischemic heart disease, heart failure, cardiac arrhythmia, diabetes, and stroke) were significantly associated with a LOS longer than 11 days $(P<0.05$, Table 2$)$.

In multivariate analysis, only admission between Thursday and Saturday, high $\mathrm{PaCO}_{2}$, low serum albumin level, and having heart failure, diabetes, or stroke were independently associated with a long LOS (Table 3). Admission between

Table 2 Results of univariate logistic regression analysis of a LOS $>$ II days versus $\leq$ II days during admission for acute exacerbation of COPD $(n=590)$

\begin{tabular}{|c|c|c|}
\hline Variables & $\begin{array}{l}\text { Odds } \\
\text { ratio }\end{array}$ & $\begin{array}{l}\text { Confidence } \\
\text { interval }\end{array}$ \\
\hline Age, years & 1.03 & $1.01-1.04$ \\
\hline Sex, female versus male & 0.97 & $0.66-1.40$ \\
\hline Pulse, beats/min & 1.01 & $1.00-1.01$ \\
\hline Long-term oxygen therapy & 1.21 & $0.60-2.43$ \\
\hline Mean arterial blood pressure, $\mathrm{mmHg}$ & 0.99 & $0.98-1.00$ \\
\hline X-ray findings consistent with pneumonia & 1.23 & $0.82-1.83$ \\
\hline Patients living in an institution & 1.28 & $0.72-2.31$ \\
\hline $\mathrm{FEV}_{1}, \mathrm{l} / \mathrm{s}$ & 0.64 & $0.42-0.97$ \\
\hline At least one COPD-related admission* & 1.66 & $1.06-2.59$ \\
\hline $\mathrm{PaCO}_{2}(\mathrm{kPa})$ & 1.25 & I.I2-I.40 \\
\hline Serum albumin level, $g / L$ & 0.93 & $0.89-0.97$ \\
\hline Admission Thursday-Saturday & 2.22 & $1.52-3.23$ \\
\hline Monday & 1.43 & $0.62-2.27$ \\
\hline Tuesday & 1.54 & $0.67-3.55$ \\
\hline Wednesday & 1.08 & $0.44-2.60$ \\
\hline Thursday & 3.97 & |.77-8.9| \\
\hline Friday & 2.19 & $0.97-4.95$ \\
\hline Saturday & 3.11 & $1.35-7.17$ \\
\hline Comorbidity, $\geq I$ versus 0 & 2.50 & $1.32-4.74$ \\
\hline Ischemic heart disease & 1.55 & $1.03-2.13$ \\
\hline Heart failure & 2.64 & $1.63-4.26$ \\
\hline Cardiac arrhythmia & 1.75 & $1.07-2.86$ \\
\hline Cancer of any form & 1.05 & $0.75-1.46$ \\
\hline Renal failure & 1.09 & $0.38-3.07$ \\
\hline Diabetes & 2.00 & $1.20-3.33$ \\
\hline Stroke & 1.91 & I.14-3.20 \\
\hline Neurologic disorders & 1.15 & $0.62-2.10$ \\
\hline Osteoporosis & 1.39 & $0.82-2.34$ \\
\hline Hypertension & 1.23 & $0.83-1.80$ \\
\hline Psychiatric diagnosis & 0.97 & $0.57-1.66$ \\
\hline Abuse of drug/alcohol & 0.72 & $0.38-1.33$ \\
\hline
\end{tabular}

Note: *At least one COPD-related admission within 12 months prior to index admission.

Abbreviations: LOS, length of stay; COPD, chronic obstructive pulmonary disease; $\mathrm{SD}$, standard deviation; $\mathrm{PaCO}_{2}$, partial pressure of carbon dioxide in arterial blood $\mathrm{FEV}_{1}$, forced expiratory volume in I second. 
Table 3 Results of multivariate logistic regression analysis of a LOS of $>$ I I days vs $\leq$ I I days during admission for AECOPD $(n=590)$

\begin{tabular}{lll}
\hline Risk factor & Odds ratio & $\mathbf{9 5 \%} \mathbf{C l}$ \\
\hline $\mathrm{PaCO}_{2}, \mathrm{kPa}$ & $\mathrm{I} .25$ & $\mathrm{I} . \mathrm{II}-\mathrm{I} .4 \mathrm{I}$ \\
Serum albumin level, g/L & $0.9 \mathrm{I}$ & $0.87-0.96$ \\
Admission Thursday-Saturday & 2.50 & $\mathrm{I} .66-3.77$ \\
Previous COPD-related admission* & $\mathrm{I} .60$ & $0.98-2.62$ \\
Comorbidities, $\geq \mathrm{I}$ versus 0 & 2.08 & $1.08-4.02$ \\
Heart failure & 2.26 & $1.34-3.80$ \\
Diabetes & 1.90 & $1.07-3.37$ \\
Stroke & 1.83 & $1.04-3.2 \mathrm{I}$ \\
\hline
\end{tabular}

Note: *At least one COPD-related admission within 12 months prior to index admission.

Abbreviations: LOS, length of stay; acute exacerbation of chronic obstructive pulmonary disease, $\mathrm{AECOPD} ; \mathrm{PaCO}_{2}$, partial pressure of carbon dioxide in arterial blood; $\mathrm{Cl}$, confidence interval.

Thursday and Saturday increased the odds ratio (OR) of a LOS longer than 11 days to 2.50 (95\% confidence interval [CI] 1.66-3.77). The OR for the presence of heart failure, diabetes, and stroke was 2.26 (95\% CI 1.34-3.80), 1.90 (95\% CI 1.07-3.37), and 1.83 (95\% CI 1.04-3.21), respectively. An increase of $1 \mathrm{kPa}$ in $\mathrm{PaCO}_{2}$ had an OR of $1.26(95 \%$ CI 1.12-1.41), and an increasing serum albumin level (OR 0.92 [95\% CI 0.88-0.97]) were associated with a shorter LOS. The area under the ROC curve was 0.73 (Figure 1).

\section{Discussion}

This study found that admission between Thursday and Saturday, high $\mathrm{PaCO}_{2}$, low serum albumin level, and having heart failure, diabetes, or stroke were independently associated with a long LOS. The statistical model had an area under the ROC curve of 0.73 , which we considered to be too low to allow the development of a meaningful predictive model.

The impact of weekday at the time of admission on length of hospital stay has hardly been studied in COPD given its impact on mortality; a large Spanish study showed that patients admitted on the weekend for AECOPD are more likely to die in the hospital compared to those admitted on weekdays. ${ }^{24}$ Our finding that admission between Thursday and Saturday was associated with a LOS greater than the 75th percentile (ie, 11 days) pointed out another possible negative consequence of being admitted around the weekend for AECOPD. Our finding is consistent with a Spanish study demonstrating an association between weekend admission (Friday-Sunday) and prolonged LOS. ${ }^{20}$ However, a long LOS was defined as $\geq 3$ days in the Spanish study, which is shorter than the median in the present study (6 days), and shorter than the commonly reported mean or median LOS of 6-11 days. ${ }^{7}$ Furthermore, over $90 \%$ of the patient population in the Spanish study was male, which stands in contrast to our patient population of more than 50\% women. Our finding, together with the finding from the Spanish study, indicates that prolonged LOS in connection to weekend admissions may be a more common phenomenon than we are aware of. This is supported by studies of other diseases that have also found that admission day of the week affects $\operatorname{LOS} .^{25,26}$ There are several possible explanations for this phenomenon, and they probably vary between specific health care systems.

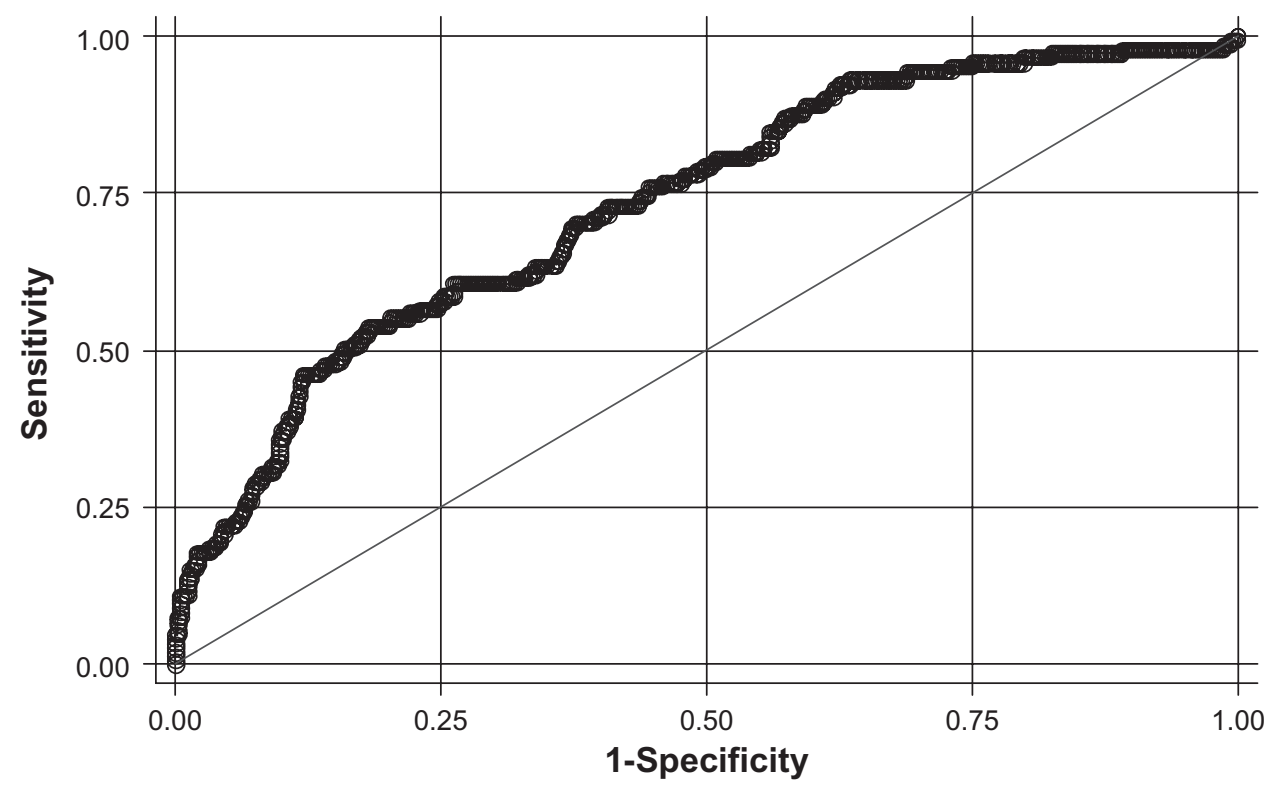

Area under ROC curve $=0.7342$

Figure I Receiver operating characteristics curve for the multivariate logistic regression model. 
In our case it might be that the "natural" discharge day of these patients was immediately before the next weekend, as indicated by the median LOS (6 days), and hence the discharge could be delayed by the discontinuity and reduction of the medical staff during weekends, as suggested by previous studies. ${ }^{16,22}$ This phenomenon might also be due to the reduced primary health care or support available from relatives following hospital discharge. Alternatively, patients admitted between Thursday and Saturday may have more severe AECOPD than patients admitted on other weekdays, as has been suggested by some authors. ${ }^{27}$

Association between comorbidity - which is common in COPD - and LOS are less studied compared with their association with mortality under/after AECOPD. The published results had either been concentrated on single diagnoses ${ }^{21,22}$ or number of comorbidities. ${ }^{11,19,20}$ In the present paper, most of the recognized comorbidities of COPD have been assessed; we found heart failure, diabetes, and stroke to be independently related with prolonged LOS. Both heart failure and diabetes are common in patients with COPD. ${ }^{28,29}$ Since the exacerbation of COPD and its treatment with beta-agonist and systemic steroids may worsen heart failure ${ }^{30}$ and diabetes, ${ }^{22}$ respectively, it is understandable that a prolonged stay might be needed to get these comorbid conditions under control.

That a high $\mathrm{PaCO}_{2}$ measured in the emergency department was associated with a prolonged LOS is reasonable because $\mathrm{PaCO}_{2}$ and other blood-gas variables reflect the severity of respiratory failure. This finding is consistent with two previous studies ${ }^{16,31}$ but inconsistent with two others. ${ }^{25,26}$ The discrepant results of these studies may be due to differences between the patient populations, since patients needing intensive care and with infiltration on chest radiographs indicating pneumonia were excluded from the latter two studies.

Our finding of an association of low serum albumin level with a long LOS is consistent with a British study demonstrating an association between a low serum albumin level and a LOS $>7$ days. ${ }^{31}$ Serum albumin level is a marker of nutritional status, ${ }^{32}$ with a low level being associated with higher longterm mortality in COPD patients. ${ }^{2,33}$ Albumin also forms a part of the acute-phase protein response, and hence low serum albumin level may reflect the deterioration of clinical status or increased persistent inflammation during AECOPD. ${ }^{33}$

In the present study, we wanted to identify patients with prolonged LOS primarily because such patients may have a worse health status and hence an increased need for post-discharge support. ${ }^{34}$ In the absence of a standardized definition of prolonged LOS, we decided to use a LOS cutoff at the 75 th percentile rather than at the median ${ }^{11-13}$ because the potential benefit from improved discharge planning would be greater with increasing LOS.

In univariate analysis, increasing age was significantly associated with a LOS longer than 11 days. However, this effect disappeared after adjusting for other variables in the multivariate analyses. This can, for example, be attributed to older patients being more fragile, having lower serum albumin values, having more comorbidities, or more severe COPD exacerbations and higher $\mathrm{PaCO}_{2}$. Therefore, age by itself was no longer significantly related to prolonged LOS, and the age effect was probably captured by the other variables.

Some evidence supports that social circumstances affect LOS. ${ }^{15}$ However, in this data set, we had no feasible variable for social factors like income, class, or (earlier) profession.

The predictive model did not perform as well as we had anticipated. The area under the ROC curve was 0.73 , suggesting a poor to fair performance. Although the usefulness of a prediction model does not follow directly from how big the area under the ROC curve, a level of $73 \%$ will in most cases be too low for the purpose of resource planning in a hospital, while a level close to $80 \%$ is usually considered sufficient for practical use. ${ }^{35}$ The present ROC curve illustrates how one must trade sensitivity for specificity - if we need to correctly identify $75 \%$ of the patients with a LOS $>11$ days, we must accept that $50 \%$ of the patients with a LOS $\leq 11$ are incorrectly classified. Alternatively, if we require that $75 \%$ of the patients with a LOS $\leq 11$ be correctly classified, only approximately $50 \%$ of the patients with a LOS $>11$ will be identified. In our opinion, these uncertainties are too large for the predictions to be useful in a clinical setting.

There may be several reasons for the suboptimal performance of this model, with one possibility being the presence of a large random component that is independent of patientrelated variables. Previous studies found that only $8 \%-12 \%$ of LOS variation could be explained by clinical variables. ${ }^{18,36}$ A US study showed that hospital stays for patients hospitalized for AECOPD were considerably extended beyond what was considered strictly necessary because physicians observed patients an extra day to be certain that patients could cope at home after discharge. ${ }^{16}$ Such individual assessment is one of several random elements that are difficult to incorporate in a statistical prediction model. Additionally, local practice guidelines, hospital resources, and the organization of care may influence LOS. ${ }^{24,37}$

Some limitations of this retrospective study should be discussed. The number of variables in the analyses was 
limited; hence, potentially important predictors might have been missed. We reduced the possibility of false-positive findings by limiting the number of variables before the analysis. Some medical records at the time of the admission were incomplete, for example, lacked information on comorbidities or results of tests actually being done on admission. This was partially compensated by imputing values for missing values for the variables that we thought would be most important $-\mathrm{FEV}_{1}, \mathrm{PaCO}_{2}$, and serum albumin level. This imputation contributed to reducing the uncertainty associated with missing data and avoided the exclusion of patients, which would have reduced the effect of the results of the study.

In our case, we had no indication that the missing values for serum albumin, $\mathrm{PaCO}_{2}$ and $\mathrm{FEV}_{1}$ were systematically missing. Therefore, imputation is reasonable. Without imputation of missing data, those patients with incomplete data would be excluded, which could also introduce bias. ${ }^{38}$

Finally, the study population was recruited from a single university hospital in Oslo, which served as a primary hospital for a geographically defined area of the city. Therefore, the external validity of our results might be limited. However, the included population was relatively large and unselected in that all patients with a discharge diagnosis of COPD were included. Furthermore, both age and LOS in this population were comparable to those in previous larger studies from other countries, which support the external validity of our sample..$^{24,31}$

\section{Conclusion}

In conclusion, we found that admission between Thursday and Saturday, $\mathrm{PaCO}_{2}$, serum albumin level, and comorbidities such as heart failure, diabetes, and stroke were associated with LOS. The reported findings may help physicians to identify patients at risk of a long LOS in the early stages of an AECOPD admission, and thus introduce the possibility of offering better follow-up. However, we were unable to create a robust scoring model for risk stratification that would have been useful for discharge planning. The reasons for prolonged LOS are probably multidimensional, and future studies should focus on both patient- and non-patientrelated factors.

\section{Acknowledgments}

This study was supported by Grant 2779038 from the South-Eastern Norway Regional Health Authority and Grant 2779010 from the Research Council of Norway.

\section{Author contributions}

YW contributed to study design, analyses and interpretation of data, drafting of the manuscript, and led data collection. KS contributed to study design, analyses and interpretation of data, and the draft and revisions of the manuscript. FAD contributed to study design, analyses and interpretation of data, and the draft and revisions of the manuscript. $\mathrm{SH}$ contributed to study design, data collection, and revisions of the manuscript. TH contributed to study design and revisions of the manuscript. All authors approved the final manuscript.

\section{Disclosure}

The authors report no conflicts of interest in this work.

\section{References}

1. Almagro P, Barreiro B, Ochoa de Echaguen A, et al. Risk factors for hospital readmission in patients with chronic obstructive pulmonary disease. Respiration. 2006;73(3):311-317.

2. Connors AF, Dawson NV, Thomas C, et al. Outcomes following acute exacerbation of severe chronic obstructive lung disease. The SUPPORT investigators (Study to Understand Prognoses and Preferences for Outcomes and Risks of Treatments). Am J Respir Crit Care Med. 1996;154(4 Pt 1):959-967.

3. Donaldson GC, Seemungal TA, Bhowmik A, Wedzicha JA. Relationship between exacerbation frequency and lung function decline in chronic obstructive pulmonary disease. Thorax. 2002;57(10):847-852.

4. Garcia-Aymerich J, Farrero E, Félez MA, Izquierdo J, Marrades RM, Antó JM; Estudi del Factors de Risc d'Agudització de la MPOC investigators. Risk factors of readmission to hospital for a COPD exacerbation: a prospective study. Thorax. 2003;58(2):100-105.

5. Seemungal TA, Donaldson GC, Paul EA, Bestall JC, Jeffries DJ, Wedzicha JA. Effect of exacerbation on quality of life in patients with chronic obstructive pulmonary disease. Am J Respir Crit Care Med. 1998;157(5 Pt 1):1418-1422.

6. Soler-Cataluña JJ, Martínez-García MA, Sánchez LS, Tordera MP, Sánchez PR. Severe exacerbations and BODE index: two independent risk factors for death in male COPD patients. Respir Med. 2009;103(5): 692-699.

7. Roche N, Rabbat A, Zureik M, Huchon G. Chronic obstructive pulmonary disease exacerbations in emergency departments: predictors of outcome. Curr Opin Pulm Med. 2010;16(2):112-117.

8. Sullivan SD, Ramsey SD, Lee TA. The economic burden of COPD. Chest. 2000;117(Suppl 2):5S-9S.

9. Chang CL, Sullivan GD, Karalus NC, Hancox RJ, McLachlan JD, Mills GD. Audit of acute admissions of chronic obstructive pulmonary disease: inpatient management and outcome. Intern Med J. 2007;37(4):236-241.

10. Díaz-Peromingo JA, Grandes-Ibáńez J, Fandińo-Orgeira JM, BarcalaVillamarín P, Garrido-Sanjuán JA. Predicting factors contributing to length of stay in hospitalized chronic obstructive pulmonary disease (COPD) patients: the role of the emergency room. Acta Medica (Hradec Kralove). 2004;47(1):29-32.

11. Incalzi RA, Pedone C, Onder G, Pahor M, Carbonin PU; GIFA. Gruppo Italiano di Farmacovigilanza (Italian Group for Pharmacological Survey in the Elderly). Predicting length of stay of older patients with exacerbated chronic obstructive pulmonary disease. Aging (Milano). 2001;13(1):49-57. 
12. BuSaba NY, Schaumberg DA. Predictors of prolonged length of stay after major elective head and neck surgery. Laryngoscope. 2007;117(10):1756-1763.

13. McDevitt J, Kelly M, Comber H, Kelleher T, Dwane F, Sharp L. A population-based study of hospital length of stay and emergency readmission following surgery for non-small-cell lung cancer. Eur $J$ Cardiothorac Surg. 2013;44(4):e253-e259.

14. Säynäjäkangas O, Kinnunen T, Tuuponen T, Keistinen T. Length of stay and interval to readmission in emergency hospital treatment of COPD. Age Ageing. 2004;33(6):567-570.

15. Wong AW, Gan WQ, Burns J, Sin DD, van Eeden SF. Acute exacerbation of chronic obstructive pulmonary disease: influence of social factors in determining length of hospital stay and readmission rates. Can Respir J. 2008;15(7):361-364.

16. Mushlin AI, Black ER, Connolly CA, Buonaccorso KM, Eberly SW. The necessary length of hospital stay for chronic pulmonary disease. JAMA. 1991;266(1):80-83.

17. Nowiński A, Kamiński D, Korzybski D, Stokłosa A, Górecka D. [The impact of comorbidities on the length of hospital treatment in patients with chronic obstructive pulmonary disease]. Pneumonol Alergol Pol. 2011;79(6):388-396. Polish.

18. Roberts CM, Lowe D, Bucknall CE, Ryland I, Kelly Y, Pearson MG. Clinical audit indicators of outcome following admission to hospital with acute exacerbation of chronic obstructive pulmonary disease. Thorax. 2002;57(2):137-141.

19. Agboado G, Peters J, Donkin L. Factors influencing the length of hospital stay among patients resident in Blackpool admitted with COPD: a cross-sectional study. BMJ Open. 2012;2(5):e000869.

20. de la Iglesia F, Valiño P, Pita S, et al. Factors predicting a hospital stay of over 3 days in patients with acute exacerbation of chronic obstructive pulmonary disease. J Intern Med. 2002;251(6):500-507.

21. Ng TP, Niti M, Tan WC, Cao Z, Ong KC, Eng P. Depressive symptoms and chronic obstructive pulmonary disease: effect on mortality, hospital readmission, symptom burden, functional status, and quality of life. Arch Intern Med. 2007;167(1):60-67.

22. Parappil A, Depczynski B, Collett P, Marks GB. Effect of comorbid diabetes on length of stay and risk of death in patients admitted with acute exacerbations of COPD. Respirology. 2010;15(6):918-922.

23. World Health Organization. International Statistical Classification of Diseases and Related Health Problems 10th Revision. Geneva: World Health Organization; 2003. Available from: http://apps.who.int/ classifications/icd10/browse/2010/en. Accessed December 20, 2011.

24. Barba R, Zapatero A, Losa JE, et al. The impact of weekends on outcome for acute exacerbations of COPD. Eur Respir J. 2012;39(1):46-50.

25. Earnest A, Chen MI, Seow E. Exploring if day and time of admission is associated with average length of stay among inpatients from a tertiary hospital in Singapore: an analytic study based on routine admission data. BMC Health Serv Res. 2006;6:6
26. Horwich TB, Hernandez AF, Liang L, et al; Get With Guidelines Steering Committee and Hospitals. Weekend hospital admission and discharge for heart failure: association with quality of care and clinical outcomes. Am Heart J. 2009;158(3):451-458.

27. van den Borst B, Wesseling G. Acute exacerbations of COPD: it's the weekend but it can't wait until Monday. Eur Respir J. 2012; 39(6): 1547.

28. Mannino DM, Thorn D, Swensen A, Holguin F. Prevalence and outcomes of diabetes, hypertension and cardiovascular disease in COPD. Eur Respir J. 2008;32(4):962-969.

29. Rutten FH, Cramer MJ, Lammers JW, Grobbee DE, Hoes AW. Heart failure and chronic obstructive pulmonary disease: An ignored combination? Eur J Heart Fail. 2006;8(7):706-711.

30. Hawkins NM, Virani S, Ceconi C. Heart failure and chronic obstructive pulmonary disease: the challenges facing physicians and health services. Eur Heart J. 2013;34(36):2795-2807.

31. Price LC, Lowe D, Hosker HS, Anstey K, Pearson MG, Roberts CM; British Thoracic Society and the Royal College of Physicians Clinical Effectiveness Evaluation Unit (CEEu). UK National COPD Audit 2003: Impact of hospital resources and organisation of care on patient outcome following admission for acute COPD exacerbation. Thorax. 2006;61(10):837-842.

32. Thorsdottir I, Gunnarsdottir I, Eriksen B. Screening method evaluated by nutritional status measurements can be used to detect malnourishment in chronic obstructive pulmonary disease. J Am Diet Assoc. 2001;101(6):648-654.

33. Gunen H, Hacievliyagil SS, Kosar F, et al. Factors affecting survival of hospitalised patients with COPD. Eur Respir J. 2005;26(2):234-241.

34. Roche N, Zureik M, Soussan D, Neukirch F, Perrotin D; Urgence BPCO (COPD Emergency) Scientific Committee. Predictors of outcomes in COPD exacerbation cases presenting to the emergency department. Eur Respir J. 2008;32(4):953-961.

35. Sicignano A, Carozzi C, Giudici D, Merli G, Arlati S, Pulici M. The influence of length of stay in the ICU on power of discrimination of a multipurpose severity score (SAPS). ARCHIDIA. Intensive Care Med. 1996;22(10):1048-1051.

36. Stolz D, Christ-Crain M, Morgenthaler NG, et al. Copeptin, C-reactive protein, and procalcitonin as prognostic biomarkers in acute exacerbation of COPD. Chest. 2007;131(4):1058-1067.

37. Kong GK, Belman MJ, Weingarten S. Reducing length of stay for patients hospitalized with exacerbation of COPD by using a practice guideline. Chest. 1997;111(1):89-94.

38. Sterne JA, White IR, Carlin JB, et al. Multiple imputation for missing data in epidemiological and clinical research: potential and pitfalls. BMJ. 2009;338:b2393.
International Journal of COPD

\section{Publish your work in this journal}

The International Journal of COPD is an international, peer-reviewed journal of therapeutics and pharmacology focusing on concise rapid reporting of clinical studies and reviews in COPD. Special focus is given to the pathophysiological processes underlying the disease, intervention programs, patient focused education, and self management protocols.

\section{Dovepress}

This journal is indexed on PubMed Central, MedLine and CAS. The manuscript management system is completely online and includes a very quick and fair peer-review system, which is all easy to use. Visi http://www.dovepress.com/testimonials.php to read real quotes from published authors. 